\title{
AN ELEMENTARY PROOF OF THE MORSE-PALAIS LEMMA FOR BANACH SPACES
}

DANG DINH ANG AND VU TRONG TUAN

Palais established the following generalization of the Morse lemma:

Theorem (Morse-Palais Lemma [1]). Let $V$ be a real Banach space, $\mathcal{O}$ a convex neighborhood of the origin and let $f: \mathcal{O} \rightarrow \boldsymbol{R}$ be a $C^{k+2}$ function $(k \geqq 1)$ having the origin as a nondegenerate critical point with $f(0)=0$. Then there is a neighborhood $U$ of the origin and a $C^{k}$ diffeomorphism: $\Phi: U \rightarrow \mathcal{O}$ with $\Phi(0)=0$ and $(D \Phi)_{0}=\mathrm{id}_{V}$ (the identity map of $V$ ) such that for $x$ in $U$, $f(\Phi(x))=\frac{1}{2}\left(D^{2} f\right)_{0}(x, x)$.

Here and henceforth, the nonexplained notations are standard and are the same as in [1]. Palais' proof of the theorem draws on the theory of differential equations. We shall give of Palais' theorem a very elementary and short proof. Our proof, which only uses a direct application of the inverse mapping theorem, is even shorter and more elementary than Palais' proof of his theorem for the Hilbert space case [2]. The idea of our proof came from a close examination of Palais' proof for the Hilbert space case (loc. cit.)

We first observe that since 0 is a nondegenerate critical point of $f$, $\left(D^{2} f\right)_{0}$ is an isomorphism of $V$ onto $V^{*}$ and $(D f)_{0}=0$.

REMARK 1. The problem is obviously equivalent to that of establishing the existence of a $C^{k}$-isomorphism $\Psi$ of an open neighborhood $U_{1}$ of 0 contained in $\mathcal{O}$ into $\mathcal{O}$, such that $\Psi(0)=0,\left(D \Psi^{\circ}\right)_{0}=\mathrm{id}_{V}$ and

$$
f(y)=\frac{1}{2}\left(D^{2} f\right)_{0}(\Psi(y), \Psi(y)), \quad y \text { in } U_{1} .
$$

For further use, we put $B=\frac{1}{2}\left(D^{2} f\right)_{0}$ and

$$
E=\{h: h \in L(V, V) \text { and } B(h(x)) y=B(h(y)) x, x \text { and } y \text { in } V\} .
$$

Our proof of the Morse-Palais lemma depends on the following two simple lemmas.

Received by the editors January 24, 1973.

AMS (MOS) subject classifications (1970). Primary 58E05.

Key words and phrases. Morse-Palais lemma for Banach spaces, nondegenerate critical point.

(c) American Mathematical Society 1973 
LemMA $1 . \quad E$ is a closed subspace of $L(V, V)$ and the map

$$
T: E \rightarrow L\left(V, V^{*}\right)
$$

defined by $T h=B \circ h$ is an isomorphism of $E$ onto $L_{s}\left(V, V^{*}\right)$.

Proof. Let $S h=B \circ h, h$ in $L(V, V)$. Then $S$ is an isomorphism of $L(V, V)$ onto $L\left(V, V^{*}\right)$ since $B$ is an isomorphism. Clearly, $T$ is the restriction of $S$ to $E$. It remains to show that $S$ maps $E$ onto $L_{s}\left(V, V^{*}\right)$.

It is clear from the definition of $E$ that $S$ maps $E$ into $L_{s}\left(V, V^{*}\right)$. Now let $C$ be in $L_{s}\left(V, V^{*}\right)$ and let $S h=C$, i.e., $B \circ h=C$. Then

$$
B(h(x)) y=C(x) y=C(y) x=B(h(y)) x .
$$

Hence $h$ is in $E$. Q.E.D.

Lemma 2. Let $\theta: E \rightarrow L_{s}\left(V, V^{*}\right)$ be the map defined by

$$
(\theta h)(x) y=B(h(x)) h(y) .
$$

Then $\theta$ is a $C^{\infty}$-isomorphism of a neighborhood of $\mathrm{id}_{V}$ onto a neighborhood of $B$ and $\theta\left(\mathrm{id}_{V}\right)=B$.

Proof. By direct computation

$$
[\theta(h+k)-\theta(h)](x) y=B(h(x)) k(y)+B(k(x)) h(y)+B(k(x))(k(y)) .
$$

Hence

$$
(D \theta)_{h}(k)(x) y=B(h(x)) k(y)+B(k(x)) h(y) .
$$

Thus $D \theta$ is linear, and hence $\theta$ is $C^{\infty}$. Furthermore

$$
(D \theta)_{\mathrm{id}_{V}} k=2 B \circ k=2 T k, \quad k \text { in } E .
$$

By Lemma $1,(D \theta)_{\mathrm{id}_{V}}$ is therefore an isomorphism, and hence, Lemma 2 follows from the inverse mapping theorem. Q.E.D.

Proof of THE Morse-Palais Lemma. By repeated applications of the fundamental theorem of calculus, and using the fact that $f(0)=0,(D f)_{0}=$ 0 , we have

Put

$$
f(y)=\int_{0}^{1} \int_{0}^{1}\left(D^{2} f\right)_{t s y} t d s d t(y) y, \quad y \text { in } \mathcal{O} .
$$

$$
G(y)=\int_{0}^{1} \int_{0}^{1}\left(D^{2} f\right)_{t s y} t d s d t .
$$

Then $G$ is a $C^{k}$-map of $\mathcal{O}$ into $L_{s}\left(V, V^{*}\right)$ and $G(0)=\frac{1}{2}\left(D^{2} f\right)_{0}=B$.

By Lemma 2, there exists an open neighborhood $W$ of $B$ which is taken by the $C^{\infty}$-map $\theta^{-1}$ onto an open neighborhood of $\operatorname{id}_{V}$. Now $G$ is in particular continuous, and hence maps an open neighborhood $U^{\prime}$ of 0 
contained in $\mathcal{O}$ into $W$. Put

$$
A(y)=\theta^{-1}(G(y)), \quad \Psi(y)=A(y) y \text { for } y \text { in } U^{\prime} .
$$

Note that $A(y)$ is in $L(V, V)$. It is readily seen that $A$ and $\Psi$ are $C^{k}$ on $U^{\prime}$, and $A(0)=\mathrm{id}_{V}$ and $\Psi(0)=0$.

Now

$$
(D \Psi)_{y}=A(y)+(D A)_{y} y, \quad(D \Psi)_{0}=A(0)=\mathrm{id}_{V} .
$$

Hence, by the inverse mapping theorem, $\Psi$ maps an open neighborhood $U_{1}$ of 0 contained in $U^{\prime}$ (hence contained in $\mathcal{O}$ ) onto an open neighborhood $U$ of 0 contained in $\mathcal{O}$. Furthermore

$$
\begin{aligned}
f(y) & =G(y)(y) y=\theta(A(y))(y) y \\
& =B(A(y)) y(A(y)) y=B(\Psi(y), \Psi(y)), \quad y \text { in } U_{1} .
\end{aligned}
$$

The theorem follows now from Remark 1 above.

\section{REFERENCES}

1. R. S. Palais, The Morse lemma for Banach spaces, Bull. Amer. Math. Soc. 75(1969), 968-971. MR 40 \#6593.

2. _ Morse theory on Hilbert manifolds, Topology 2 (1963), 299-340. MR 28 \#1633.

Faculty of Science and faculty of Pedagogy, University of Saigon, Saigon, VIETNAM

Current address: 57 Duy-Tan, Saigon, Vietnam 